\title{
Constant Proportion Portfolio Insurance in presence of Jumps in Asset Prices
}

\author{
Rama Cont \\ Center for Financial Engineering, \\ Columbia University, New York \\ and \\ CMAP - Ecole Polytechnique, France. \\ E-mail: Rama.Cont@columbia.edu
}

\author{
Peter Tankov \\ Laboratoire de Probabilités et \\ Modèles Aléatoires \\ Université Paris VII \\ e-mail: tankov@math.jussieu.fr \\ (corresponding author)
}

February 7, 2007

\begin{abstract}
Constant proportion portfolio insurance (CPPI) allows an investor to limit downside risk while retaining some upside potential by maintaining an exposure to risky assets equal to a constant multiple $m>1$ of the cushion, the difference between the current portfolio value and the guaranteed amount. In diffusion models with continuous trading, this strategy has no downside risk, whereas in real markets this risk is nonnegligible and grows with the multiplier value. We study the behavior of CPPI strategies in models where the price of the underlying portfolio may experience downward jumps. This allows to quantify the "gap risk" of the portfolio while maintaining the analytical tractability of the continuous-time framework. We establish a direct relation between the value of the multiplier $m$ and the risk of the insured portfolio, which allows to choose the multiplier based on the risk tolerance of the investor, and provide a Fourier transform method for computing the distribution of losses and various risk measures (VaR, expected loss, probability of loss) over a given time horizon. The results are applied to a jump-diffusion model with parameters estimated from market data.
\end{abstract}

Key words: Portfolio insurance, CPPI, Lévy process, Value at Risk, expected loss.

MSC Classification (2000) : 60G51, 91B28, 91B30. 


\section{Contents}

1 Introduction 3

1.1 Constant Proportion Portfolio Insurance . . . . . . . . . . . . . 3

1.2 Price jumps and "gap risk" . . . . . . . . . . . . . . . 4

2 Model setup 5

3 Measuring gap risk for CPPI strategies $\quad 7$

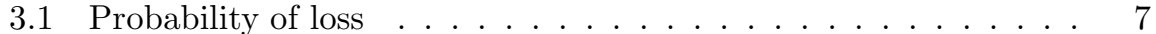

3.2 Expected loss . . . . . . . . . . . . . . . . . 7

3.3 Loss distribution . . . . . . . . . . . . . . . . . . . 9

4 A jump-diffusion example $\quad 10$

5 Discussion 14 


\section{Introduction}

The term portfolio insurance refers to portfolio management techniques designed to guarantee that the portfolio value at maturity or up to maturity will be greater or equal to a given lower bound (floor), typically fixed as a percentage of the initial investment. These techniques allow the investor to limit downside risk while retaining some potential in case of an upside market move which is however reduced in comparison with the unprotected portfolio (see [8] for a comparison of cost of different portfolio insurance strategies).

One popular method (option based portfolio insurance - OBPI) [7, 12] is to combine a position in the risky asset with a (put) option on this asset. In this paper we are interested in another widely used strategy which, contrary to OBPI, does not use options and is therefore simpler to implement: the constant proportion portfolio insurance (CPPI), popularized by Black and Jones [2] and Perold $[3,13]$. This strategy is based on the notion of cushion, that is the difference between the fund value and the floor. An amount of money proportional to the cushion is invested into the risky asset - typically an index or a portfolio of stocks - and the remainder is used to buy riskless bonds. The exposure to the risky asset is thus gradually reduced when the markets move down and the portfolio value approaches the floor.

\subsection{Constant Proportion Portfolio Insurance}

The CPPI strategy is a self-financing strategy whose goal is to guarantee a fixed amount $N$ of capital at maturity $T$. To achieve this, at any date $t$, a fraction of the portfolio is invested into the risky asset $S_{t}$ and the remainder is invested into zero-coupon bond with maturity $T$ and nominal $N$, whose price is denoted by $B_{t}$. Denoting the value of the fund by $V_{t}$,

- if $V_{t}>B_{t}$, the risky asset exposure (amount of money invested into the risky asset) is given by $m C_{t} \equiv m\left(V_{t}-B_{t}\right)$, where $C_{t}$ is the 'cushion' and $m>1$ is a constant multiplier.

- if $V_{t} \leq B_{t}$, the entire portfolio is invested into the zero-coupon.

Assume first that the interest rate $r$ is constant and that the underlying asset follows the Black-Scholes model

$$
\frac{d S_{t}}{S_{t}}=r d t+\sigma d W_{t}
$$

Then it is easy to see from the definition of the strategy that the cushion also satisfies the Black-Scholes SDE

$$
\frac{d C_{t}}{C_{t}}=(m \mu+(1-m) r) d t+m \sigma d W_{t}
$$

which is solved explicitly by

$$
C_{T}=C_{0} \exp \left(r T+m(\mu-r) T+m \sigma W_{T}-\frac{m^{2} \sigma^{2} T}{2}\right) .
$$


and hence

$$
V_{T}=N+\left(V_{0}-N e^{-r T}\right) \exp \left(r T+m(\mu-r) T+m \sigma W_{T}-\frac{m^{2} \sigma^{2} T}{2}\right)
$$

This means that in the Black-Scholes model with continuous trading, the CPPI strategy is equivalent to taking a long position in a zero-coupon bond with nominal $N$ to guarantee the capital at maturity and investing the remaining sum into a (fictitious) risky asset which has $m$ times the excess return and $m$ times the volatility of $S$ and is perfectly correlated with $S$.

\subsection{Price jumps and "gap risk"}

Formula (1) shows that in the Black-Scholes model with continuous trading there is no risk of going below the floor, regardless of the multiplier value. On the other hand the expected return of a CPPI-insured portfolio is

$$
E\left[V_{T}\right]=N+\left(V_{0}-N e^{-r T}\right) \exp (r T+m(\mu-r) T) .
$$

We then arrive to a paradoxical conclusion that in the Black-Scholes model, whenever $\mu>r$, the expected return of a CPPI portfolio can be increased indefinitely and without risk, by taking a high enough multiplier.

In reality, the possibility of reaching the floor, known as "gap risk", is widely recognized by CPPI managers: there is a nonzero probability that, during a sudden downside move, the fund manager will not have time to readjust the portfolio, which then goes crashing through the floor. Beyond the (widely documented) econometric issue of whether jumps occur or not in a given asset's price, a fundamental point is one of liquidity of the underlying: many CPPI strategies are written on funds which may be thinly traded, leading to jumps in the market price due to liquidity effects.

Since the volatility of $V_{t}$ is proportional to $m$, the risk of such loss increases with $m$, and in practice, the value of $m$ should be fixed by relating it to an acceptance threshold for the probability of loss or some other risk measure.

One approach suggested in the literature [1] is to drop the continuous trading hypothesis and use the large deviations theory to estimate the possible losses between two consecutive trading dates. However the frequency of trading interventions during a downside market move is hard to predict, making this parameter difficult to determine.

We study the behavior of CPPI strategies in models where the price of the underlying portfolio may experience downward jumps. This allows to quantify in a meaningful manner the "gap risk" of the portfolio while maintaining the analytical tractability of the continuous-time framework. We establish a direct relation between the value of the multiplier $m$ and the risk of the insured portfolio, which allows to choose the multiplier based on the risk tolerance of the investor, and provide a Fourier transform method for computing the distribution of losses and various risk measures (VaR, expected loss, probability of loss) over a given time horizon. The original financial problem (relating the risk to 
the value of the multiplier) is thus easier to solve in a model with jumps than in the classical Black-Scholes model.

Introducing price jumps to quantify the risk of CPPI strategies is quite natural. In fact, to hedge against downward jumps, some CPPI managers have issued "Crash notes", paying on a quarterly basis a spread above LIBOR under the condition that the value of the underlying portfolio does not drop by more than, say, $5 \%$, in which case only a fraction of the notional is refunded to the holder of the note.

CPPI strategies in presence of jumps in stock prices were also considered in [14]. With respect to their study, we consider various risk measures for the loss and provide analytical methods to compute them.

Finally, CPPI strategies are increasingly applied to credit portfolios. Credit CPPI products, such as BNP Paribas DYNAMO notes issued in 2005, are based on CPPI-type strategies applied to a portfolio of defaultable bonds or credit default swaps. In this case the underlying portfolio naturally experiences downward jumps at each default event and the continuity assumption is simply not tenable.

\section{Model setup}

We suppose that the price processes for the risky asset $S$ and for the zero-coupon $B$ may be written as

$$
\frac{d S_{t}}{S_{t-}}=d Z_{t} \quad \text { and } \quad \frac{d B_{t}}{B_{t-}}=d R_{t}
$$

where $Z$ and $R$ are semimartingales. As a simple example, one can take $Z$ a Lévy process and $R_{t}=r T$ for some constant interest rate $r$. A less trivial example of $\left(B_{t}\right)$ is provided by the Vasicek model.

Example 1. The Vasicek model is a one-factor interest rate model where the short rate $r_{t}$ follows (under the risk-neutral probability) an Ornstein-Uhlenbeck process:

$$
d r_{t}=\left(\alpha-\beta r_{t}\right) d t+\sigma d W_{t}
$$

The zero coupon is given by

$$
B_{t}=B(t, T)=E\left[e^{-\int_{t}^{T} r_{s} d s}\right] .
$$

It follows that in the Vasicek model the zero-coupon satisfies the stochastic differential equation

$$
\frac{d B_{t}}{B_{t}}=r_{t} d t-\sigma \frac{1-e^{-\beta(T-t)}}{\beta} d W_{t} .
$$

We make the following hypotheses:

- $\Delta Z_{t}>-1$ almost surely. 
- The zero-coupon price process $B$ is continuous.

While the first hypothesis guarantees the positivity of the risky asset price, the second one allows to focus on the impact of jumps in the underlying asset. This assumption implies in particular

$$
B_{t}=B_{0} \exp \left(R_{t}-\frac{1}{2}[R]_{t}\right)>0 \quad \text { a.s. }
$$

Let $\tau=\inf \left\{t: V_{t} \leq B_{t}\right\}$. Since the CPPI strategy is self-financing, up to time $\tau$ the portfolio value satisfies

$$
d V_{t}=m\left(V_{t-}-B_{t}\right) \frac{d S_{t}}{S_{t-}}+\left\{V_{t-}-m\left(V_{t-}-B_{t}\right)\right\} \frac{d B_{t}}{B_{t}},
$$

which can be rewritten as

$$
\frac{d C_{t}}{C_{t-}}=m d Z_{t}+(1-m) d R_{t},
$$

where $C_{t}=V_{t}-B_{t}$ denotes the cushion.

Change of numeraire Introducing the discounted cushion $C_{t}^{*}=\frac{C_{t}}{B_{t}}$ and applying Itô formula to this process, we find

$$
\frac{d C_{t}^{*}}{C_{t-}^{*}}=m\left(d Z_{t}-d[Z, R]_{t}-d R_{t}+d[R]_{t}\right),
$$

and from now on we will write

$$
L_{t} \equiv Z_{t}-[Z, R]_{t}-R_{t}+[R]_{t} .
$$

Equation (2) can then be rewritten compactly as

$$
C_{t}^{*}=C_{0}^{*} \mathcal{E}(m L)_{t},
$$

After time $\tau$, according to our definition of the CPPI strategy, the process $C^{*}$ remains constant. Therefore, the discounted cushion value for this strategy can be written explicitly as

$$
C_{t}^{*}=C_{0}^{*} \mathcal{E}(m L)_{t \wedge \tau},
$$

or alternatively

$$
\frac{V_{t}}{B_{t}}=1+\left(\frac{V_{0}}{B_{0}}-1\right) \mathcal{E}(m L)_{t \wedge \tau} .
$$

Since the stochastic exponential can become negative, in presence of negative jumps of sufficient size in the stock price, the capital $N$ at maturity is no longer guaranteed by this strategy. 


\section{Measuring gap risk for CPPI strategies}

\subsection{Probability of loss}

A CPPI-insured portfolio incurs a loss (breaks through the floor) if, for some $t \in[0, T], V_{t} \leq B_{t}$. The event $V_{t} \leq B_{t}$ is equivalent to $C_{t}^{*} \leq 0$ and since $R$ is continuous and $\mathcal{E}(X)_{t}=\mathcal{E}(X)_{t-}\left(1+\Delta X_{t}\right), C_{t}^{*} \leq 0$ for some $t \in[0, T]$ if and only if $m \Delta L_{t} \leq-1$ for some $t \in[0, T]$. This leads us to the following result:

Proposition 1. Let $L$ be of the form $L=L^{c}+L^{j}$, where $L^{c}$ is a continuous process and $L^{j}$ is an independent Lévy process with Lévy measure $\nu$. Then the probability of going below the floor is given by

$$
P\left[\exists t \in[0, T]: V_{t} \leq B_{t}\right]=1-\exp \left(-T \int_{-\infty}^{-1 / m} \nu(d x)\right) .
$$

Proof. This result follows from the fact that the number of jumps of the Lévy process $L^{j}$ in the interval $[0, T]$, whose sizes fall in $(-\infty,-1 / m]$ is a Poisson random variable with intensity $T \nu((-\infty,-1 / m])$.

Corollary 1. Assume that $S$ follows an exponential Lévy model of the form

$$
S_{t}=S_{0} e^{N_{t}}
$$

where $N$ is a Lévy process with Lévy measure $\nu$. Then the probability of going below the floor is given by

$$
P\left[\exists t \in[0, T]: V_{t} \leq B_{t}\right]=1-\exp \left(-T \int_{-\infty}^{\log (1-1 / m)} \nu(d x)\right)
$$

Proof. It follows from proposition 3 that there exists another Lévy process $L$ satisfying

$$
\frac{d S_{t}}{S_{t}}=d L_{t}
$$

The Lévy measure of $L$ is given by

$$
\tilde{\nu}^{L}(A)=\int 1_{A}\left(e^{x}-1\right) \nu(d x) .
$$

Applying proposition 1 finishes the proof.

\section{$3.2 \quad$ Expected loss}

We now study the distribution of loss of a CPPI-managed portfolio given that a loss occurs, with the aim of computing its expectation and other functionals (risk measures).

To obtain some explicit formulae, we assume that the process $L$ appearing in the stochastic exponential in (3) is a Lévy process, and we denote its Lévy 
measure by $\nu$. We can always write $L=L^{1}+L^{2}$ where $L^{2}$ is a process with piecewise constant trajectories and jumps satisfying $\Delta L_{t}^{2} \leq-1 / m$ and $L^{1}$ is a process with jumps satisfying $\Delta L_{t}^{1}>-1 / m$. In other words, $L^{1}$ has Lévy measure $\nu(d x) 1_{x>-1 / m}$ and $L^{2}$ has Lévy measure $\nu(d x) 1_{x \leq-1 / m}$, no diffusion component and no drift. Denote by $\lambda^{*}:=\nu((-\infty,-1 / m])$ the jump intensity of $L^{2}$, by $\tau$ the time of the first jump of $L^{2}$ (it is an exponential random variable with intensity $\lambda^{*}$ ) and by $\tilde{L}^{2}=\Delta L_{\tau}^{2}$ the size of the first jump of $L^{2}$. Let $\phi_{t}$ be the characteristic function of the Lévy process $\log \mathcal{E}\left(m L^{1}\right)_{t}$ and $\psi(u)=$ $\frac{1}{t} \log \phi_{t}(u)$. Finally, we suppose without loss of generality that the discounted cushion satisfies $C_{0}^{*}=1$.

First we compute the expectation of loss given that a loss occurs.

Proposition 2. Assume

$$
\int_{1}^{\infty} x \nu(d x)<\infty
$$

Then

$$
E\left[C_{T}^{*} \mid \tau \leq T\right]=\frac{\lambda^{*}+m \int_{-1}^{-1 / m} x \nu(d x)}{\left(1-e^{-\lambda^{*} T}\right)\left(\psi(-i)-\lambda^{*}\right)}\left(e^{-\lambda^{*} T} \phi_{T}(-i)-1\right) .
$$

Proof. The discounted cushion satisfies

$$
C_{T}^{*}=\mathcal{E}\left(m L^{1}\right)_{\tau \wedge T}\left(1+m \tilde{L}^{2} 1_{\tau \leq T}\right)=\mathcal{E}\left(m L^{1}\right)_{T} 1_{\tau>T}+\mathcal{E}\left(m L^{1}\right)_{\tau}\left(1+m \tilde{L}^{2}\right) 1_{\tau \leq T} .
$$

Since $L^{1}$ and $L^{2}$ are Lévy processes, $\tau, \tilde{L}^{2}$ and $L^{1}$ are independent. Since by [15, Theorem 25.17], and by definition of $\phi_{t}$,

$$
E\left[\mathcal{E}\left(m L^{1}\right)_{t}\right]=\phi_{t}(-i)
$$

we have

$$
\begin{aligned}
E\left[C_{T}^{*} \mid \tau \leq T\right] & =\frac{E\left[1+m \tilde{L}^{2}\right]}{1-e^{-\lambda^{*} T}} \int_{0}^{T} \lambda^{*} e^{-\lambda^{*} t} E\left[\mathcal{E}\left(m L^{1}\right)_{t}\right] d t \\
& =\left(\lambda^{*}+m \int_{-1}^{-1 / m} x \nu(d x)\right) \frac{1}{1-e^{-\lambda^{*} T}} \int_{0}^{T} e^{-\lambda^{*} t} \phi_{t}(-i) d t .
\end{aligned}
$$

and the result follows.

Remark 1. Suppose that $\int_{\mathbb{R}}|x| \nu(d x)<\infty$ and let $\left(\sigma^{2}, \nu, \gamma\right)$ be the characteristic triplet of $L$ with respect to zero truncation function (general Lévy measures may be treated along the same lines with a slightly heavier notation). Proposition 3 and the Lévy-Khintchine representation then give the characteristic exponent of $\log \mathcal{E}(m L)_{t}$ :

$$
\begin{aligned}
\psi(u) & =-\frac{m^{2} \sigma^{2} u^{2}}{2}+i u\left(m \gamma-\frac{\sigma^{2} m^{2}}{2}\right)+\int_{z>-1 / m}\left(e^{i u \log (1+m z)}-1\right) \nu(d z) \\
\psi(-i) & =m \gamma+m \int_{z>-1 / m} z \nu(d z) .
\end{aligned}
$$


From equation (5) it follows that the expected gain conditional on the fact that the floor is not broken satisfies

$$
E\left[C_{T}^{*} \mid \tau>T\right]=E\left[\mathcal{E}\left(m L^{1}\right)_{T}\right]=\phi_{T}(-i)=\exp \left\{T m \gamma+T m \int_{z>-1 / m} z \nu(d z)\right\} .
$$

Therefore, similarly to the Black-Scholes case, conditional expected gain in an exponential Lévy model is increasing with the multiplier, provided the underlying Lévy process has a positive expected return.

\subsection{Loss distribution}

For computing risk measures, we need the distribution function of the loss given that a loss occurred, that is, we want to compute, for $x<0$, the quantity

$$
P\left[C_{T}^{*}<x \mid \tau \leq T\right] .
$$

Our approach for computing this conditional distribution function is to express its characteristic function explicitly in terms of the characteristic exponents of the Lévy processes involved and recover the distribution function by numerical Fourier inversion. A similar strategy was used in $[4,11]$ for pricing European options. In the theorem below,

$$
\tilde{\phi}:=\frac{1}{\lambda^{*}} \int_{-\infty}^{-1 / m} e^{i u \log (-1-m x)} \nu(d x)
$$

denotes the characteristic function of $\log \left(-1-m \tilde{L}^{2}\right)$.

Theorem 1. Choose a random variable $X^{*}$ with characteristic function $\phi^{*}$, such that $E\left[\left|X^{*}\right|\right]<\infty$ and $\frac{\left|\phi^{*}(u)\right|}{1+|u|} \in L^{1}$. If

$$
\begin{aligned}
& \frac{|\tilde{\phi}(u)|}{(1+|u|)\left|\lambda^{*}-\psi(u)\right|} \in L^{1} \\
& \int_{\mathbb{R} \backslash[-\varepsilon, \varepsilon]}|\log | 1+m x|| \nu(d x)<\infty
\end{aligned}
$$

for sufficiently small $\varepsilon$, then for every $x<0$,

$$
\begin{aligned}
& P\left[C_{T}^{*}<x \mid \tau \leq T\right]=P\left[-e^{X^{*}}<x\right] \\
& \quad+\frac{1}{2 \pi} \int_{\mathbb{R}} e^{-i u \log (-x)}\left(\frac{\lambda^{*} \tilde{\phi}(u)}{i u\left(\lambda^{*}-\psi(u)\right)} \frac{1-e^{-\lambda^{*} T+\psi(u) T}}{1-e^{-\lambda^{*} T}}-\frac{\phi^{*}(u)}{i u}\right) d u .
\end{aligned}
$$

Remark 2 . The random variable $X^{*}$ is needed only to regularize the characteristic function of the loss distribution, since its cumulative distribution function is not integrable. In practice, $X^{*}$ can always be taken equal to a standard normal random variable. 
Proof. From equation (5) it follows that the characteristic function of $\log \left(-C_{T}^{*}\right)$ conditionally on the fact that a loss occurs, satisfies

$$
\begin{aligned}
E\left[e^{i u \log \left(-C_{T}^{*}\right)} \mid \tau \leq T\right] & =\frac{1}{1-e^{-\lambda^{*} T}} \int_{0}^{T} \lambda^{*} e^{-\lambda^{*} t} E\left[e^{i u \log \left(-\mathcal{E}\left(m L^{1}\right)_{t}\left(1+m \tilde{L}^{2}\right)\right)}\right] d t \\
& =\frac{1}{1-e^{-\lambda^{*} T}} \int_{0}^{T} \lambda^{*} e^{-\lambda^{*} t} e^{t \psi(u)} \tilde{\phi}(u) d t \\
& =\frac{\tilde{\phi}(u)\left(1-e^{-\lambda^{*} T+\psi(u) T}\right)}{\left(\lambda^{*}-\psi(u)\left(1-e^{-\lambda^{*} T}\right)\right.} .
\end{aligned}
$$

The integral in (9) converges at $u \rightarrow \infty$ due to the theorem's conditions and the fact that

$$
\left|\frac{1-e^{-\lambda^{*} T+\psi(u) T}}{1-e^{-\lambda^{*} T}}\right|<\frac{1+e^{-\lambda^{*} T}}{1-e^{-\lambda^{*} T}},
$$

On the other hand, condition (8) is equivalent to

$$
\begin{aligned}
& E\left[\left|\log \left(-1-m \tilde{L}^{2}\right)\right|\right]<\infty \\
& E\left[\left|\log \mathcal{E}\left(m L^{1}\right)_{T}\right|\right]<\infty,
\end{aligned}
$$

and together with the hypotheses $E\left[\left\|X^{*}\right\|\right]<\infty$, this proves that $\phi(u)=1+$ $O(u), \tilde{\phi}(u)=1+O(u)$ and $\phi^{*}(u)=1+O(u)$ as $u \rightarrow 0$, and therefore the integrand in (9) is bounded and therefore integrable in the neighborhood of zero. The proof is completed by applying Lemma 1.

\section{A jump-diffusion example}

Let us now illustrate the results of section 3 in the case of Kou's jump diffusion model [10] with parameters estimated from daily returns of a stock and a market index.

Presentation of the model and parameter estimation The Kou model is an exponential Lévy model where the driving Lévy process has a non-zero Gaussian component and a Lévy density of the form

$$
\nu(x)=\frac{\lambda(1-p)}{\eta_{+}} e^{-x / \eta_{+}} 1_{x>0}+\frac{\lambda p}{\eta_{-}} e^{-|x| / \eta_{-}} 1_{x<0} .
$$

Here, $\lambda$ is the total intensity of positive and negative jumps, $p$ is the probability that a given jump is negative and $\eta_{-}$and $\eta_{+}$are characteristic lengths of respectively negative and positive jumps.

To estimate the model from market data, we use the empirical characteristic function as suggested in $[16,17]$ : we find the parameter vector $\theta=$ $\left(b, \sigma, \lambda, p, \eta_{+}, \eta_{-}\right)$by minimizing

$$
\int_{-K}^{K}\left|\psi_{\theta}(u)-\hat{\psi}(u)\right|^{2} w(u) d u .
$$




\begin{tabular}{l|cccccc} 
Series & $\mu$ & $\sigma$ & $\lambda$ & $p$ & $\eta_{+}$ & $\eta_{-}$ \\
\hline MSFT & -0.11 & 0.257 & 83.5 & 0.34 & 0.0209 & 0.0262 \\
GM & -0.518 & 0.271 & 76.9 & 0.243 & 0.0166 & 0.0240
\end{tabular}

Table 1: Kou model parameters estimated from MSFT and GM time series.

where

$$
\hat{\psi}(u)=\frac{1}{t} \log \frac{1}{N} \sum_{k=1}^{N} e^{i u X_{i}}
$$

is the empirical characteristic exponent,

$$
\psi_{\theta}(u)=-\frac{\sigma^{2} u^{2}}{2}+i \gamma u+\frac{\lambda p}{1+i u \eta_{-}}+\frac{\lambda(1-p)}{1-i u \eta_{+}}-\lambda
$$

is the characteristic exponent of the Kou model and

$$
w(u)=\frac{1}{1+\alpha u^{2}}
$$

is the weight function used to attribute more weights to low frequencies and thus to the tails of the distribution. The tuning parameter values were fixed to $\alpha=0.01$ and $K=50$ based on tests with simulated data.

The parameters of Kou's jump diffusion model were estimated for daily time series of the General Motors Corporation (GM) and of the Microsoft Corporation (MSFT) share prices. We used 10 years of data, from December 1st 1996 to December 1st 2006, making a total of 2500 data points for each series. The estimated parameter values are shown in table 1 . Figure 1 shows that Kou model fits the smoothed returns density quite well, in particular, the exponential tail decay seems to be a realistic assumption.

Loss probability Assuming the (discounted) risky asset price process follows the Kou exponential Lévy model, equation (4) for loss probability yields

$$
P\left[\exists t \in[0, T]: V_{t} \leq B_{t}\right]=1-\exp \left(-T p \lambda(1-1 / m)^{1 / \eta_{-}}\right) .
$$

Figure 2 shows the dependence of the loss probability on the multiplier for a CPPI portfolios containing MSFT and GM stocks as risky assets. Although Microsoft is slightly riskier, the loss probabilities for the two stocks are quite similar. A $5 \%$ loss probability over 5 years corresponds to a multiplier value of about 5.5 for Microsoft and 6 for General Motors.

Expected loss Once again, suppose that the discounted risky asset

$$
S_{t}^{*}=\mathcal{E}(L)_{t}
$$

follows the Kou exponential Lévy model

$$
S_{t}^{*}=S_{0}^{*} e^{N_{t}}
$$




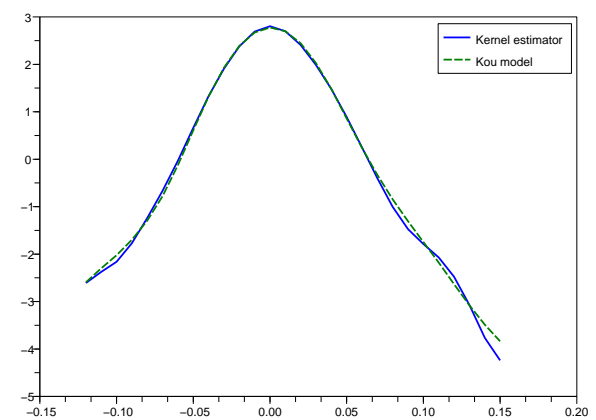

Figure 1: Logarithm of the density for MSFT time series. Solid line: kernel density estimator. Dashed line: Kou model with parameters estimated via empirical characteristic function.

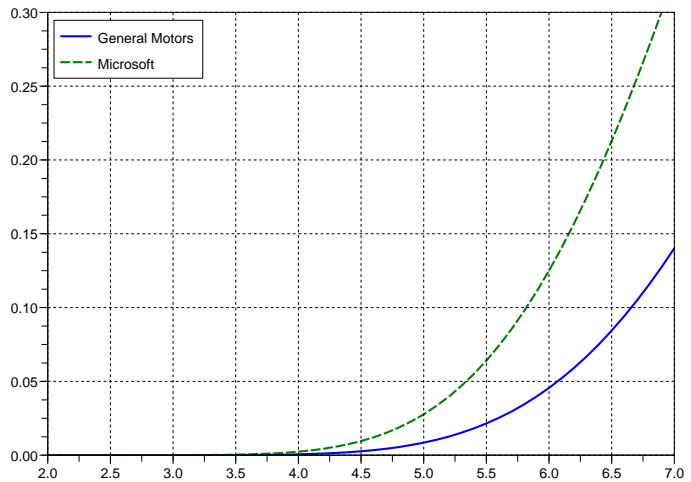

Figure 2: Probability of loss as a function of the multiplier. 
where $N$ is a Lévy process with volatility $\sigma$, drift $b$ and Lévy density $\nu$ of the form (10). For convenience of notation we write $\lambda_{ \pm}=1 / \eta_{ \pm} ; c_{-}=p \lambda$; $c_{+}=(1-p) \lambda$.

We suppose $\lambda_{+}>1$. Proposition 3 implies that $L$ is a Lévy process with volatility $\sigma$, drift $b+\frac{\sigma^{2}}{2}$ and Lévy density

$$
\nu_{L}(x)=\lambda_{+} c_{+}(1+x)^{-1-\lambda_{+}} 1_{x>0}+\lambda_{-} c_{-}(1+x)^{-1+\lambda_{-}} 1_{-1<x<0} .
$$

Splitting this Lévy density in two parts, we find on one hand

$$
\begin{aligned}
\lambda^{*} & =c_{-}(1-1 / m)^{\lambda_{-}}, \\
1+\frac{m}{\lambda^{*}} \int_{-1}^{-1 / m} x \nu_{L}(d x) & =-\frac{m-1}{\lambda_{-}+1},
\end{aligned}
$$

and on the other hand, from (6), the characteristic exponent of the Lévy process $\log \mathcal{E}\left(m L^{1}\right)_{t}$ at the point $-i$ is

$$
\begin{aligned}
\psi(-i)=m\left(b+\sigma^{2} / 2\right)+\frac{c_{+} m}{\lambda_{+}-1}-\frac{c_{-} m}{\lambda_{-}+1} & \\
& \quad-\frac{c_{-} \lambda_{-} m(1-1 / m)^{\lambda_{-}+1}}{\lambda_{-}+1}+c_{-} m(1-1 / m)^{\lambda_{-}} .
\end{aligned}
$$

Finally, assembling the two factors we get the expected loss:

$$
E\left[C_{T}^{*} \mid \tau \leq T\right]=-\frac{(m-1)\left(1-e^{-\lambda^{*} T+\psi(-i) T}\right) \lambda^{*}}{\left(\lambda_{-}+1\right)\left(1-e^{-\lambda^{*} T}\right)\left(\lambda^{*}-\psi(-i)\right)} .
$$

Figure 4 shows the dependence of the conditional (on the fact that a loss occurs) and the unconditional expected loss of a CPPI portfolio with MSFT and GM stocks as risky assets for a time horizon of $T=3$ years. While the loss probability for the two CPPI funds is roughly the same, the MSFT-based fund has a much bigger expected loss, almost matching the initial investment for $m=5$. This happens because the MSFT stock has a bigger expected return (about 30\% per year compared to around zero for GM). Before the loss-making jump, the MSFT-based fund will have a better performance, leading to a bigger proportion of risky asset in the portfolio and therefore a bigger loss after a large negative jump. Of course, not only the expected loss but also the expected gain of the MSFT-based portfolio will be much bigger than that of GM-based one.

Loss distribution In the case of a general Kou's model, the integrand in (9) must be evaluated numerically. Therefore, for illustration purposes we consider here a particular case of Kou model with $p=1$ (only negative jumps) and $\eta_{-}=1$. This amounts to saying that

$$
L_{t}=\mu t+\sigma W_{t}+\sum_{i=1}^{N_{t}} Y_{i}
$$



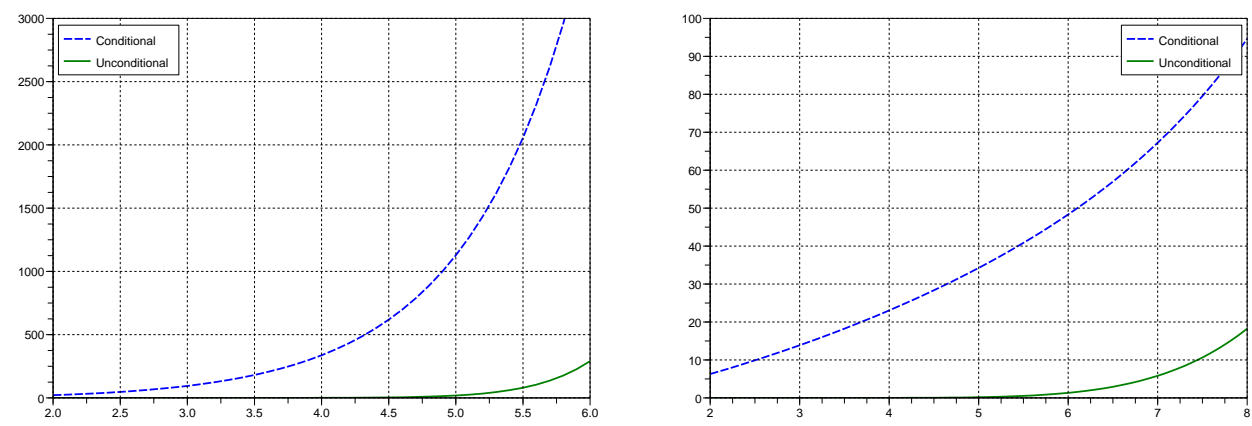

Figure 3: Expected loss over $T=3$ years as a function of the multiplier, for nominal $N=1000 \$$ and $r=0.04$. Left: MSFT; Right: GM.

where $N$ is a Poisson process with intensity $\lambda$ and $\left\{Y_{i}\right\}$ are independent uniforms on $[-1,0]$. That is, we suppose that during a crash the jump size distribution is uniform on $[-1,0]$. It is easy to check that the condition (8) is satisfied. This model can describe, for example, a defaultable asset with random recovery on the interval $[0,1]$ in case of default. In this case, $\lambda^{*}=\lambda(1-1 / m)$ and $\tilde{L}^{2} \sim U([-1,-1 / m])$. An easy computation then shows that

$$
\tilde{\phi}(u)=E\left[e^{i u \tilde{L}^{2}}\right]=\frac{(m-1)^{i u}}{1+i u},
$$

hence, condition (7) is also satisfied. On the other hand,

$$
\log \mathcal{E}\left(m L^{1}\right)_{t}=m(\mu-r) t+m \sigma W_{t}+\sum_{i=1}^{N_{t}} \log \left(1+m \Delta L_{t}^{1}\right),
$$

and therefore

$$
\psi(u)=i u\left\{m(\mu-r)-\frac{m^{2} \sigma^{2}}{2}\right\}-\frac{u^{2} m^{2} \sigma^{2}}{2}-\frac{\lambda}{m} \frac{i u}{1+i u} .
$$

We see that the expression under the integral in (9) is explicit and only the final integration must be done numerically.

Figure 4 shows the (unconditional) distribution of losses in this example, with data $\mu=0.1, \sigma=0.2, r=0.03, \lambda=1 / 3$. The initial capital was $N=1000 \$$, the time horizon $T=2$ years and the multiplier $m=2$. The $5 \%$ probability corresponds approximately to a loss size of $62 \$$ (this is the $5 \% \mathrm{VaR}$ ).

\section{Discussion}

We have proposed a simple framework for studying the "gap risk" of CPPI strategies, caused by downward jumps in the value of the underlying portfolio. 


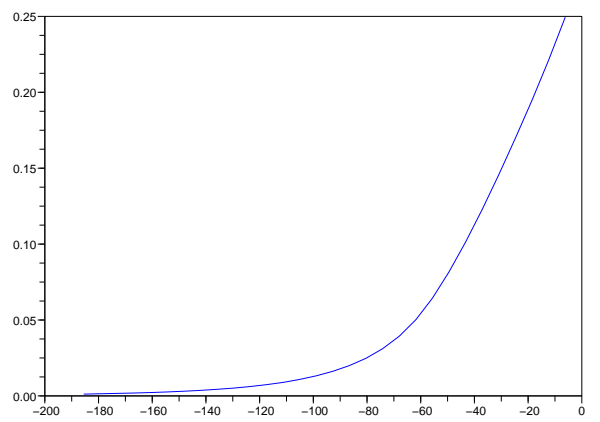

Figure 4: Probability of loss of a given size as a function of loss size (distribution function of losses).

Our study shows that jump risk is not only significant for CPPI strategies but also leads to a criterion for adjusting the multiplier based on the investor's risk aversion. Our framework leads to analytically tractable expressions for the probability of hitting the floor, the expected loss and the distribution of losses.

We have illustrated these computations in the case of a jump-diffusion model, with parameters estimated on daily stock returns. While these data reveal a relatively low level of jump risk, they are not necessarily the ones to use from a risk management perspective: for a CPPI strategy, the choice of jump parameters can be used to design a stress test of the strategy and the values of jump parameters can be determined with this interpretation in mind.

A natural question is to compute the cost of hedging this gap risk. As mentioned earlier, this is sometimes done by using "crash notes". The values of spreads paid for such notes (typically 100 bps above LIBOR for many CPPI funds) can also be used as an indicator of "risk-neutral" jump parameters which can be used for pricing the gap risk.

Another interesting extension is to consider, in the present framework, the valuation of options on CPPI funds: such options are currently traded in the markets. In this case, we are dealing with options on an underlying whose price can experience jumps: since jump risk will play an important role for the CPPI fund, our previous study [6] suggest that delta-hedging may not be the best choice and other hedging strategies can be more efficient.

We hope to address these issues in a forthcoming work. 


\section{Appendix A}

In this appendix, we recall two results from stochastic calculus. Details and proofs may be found in [5, Chapter 9] or [9].

Let $X$ be a semimartingale. Then the stochastic differential equation

$$
\frac{d Y_{t}}{Y_{t-}}=d X_{t}, \quad Y_{0}=1,
$$

has a unique strong solution called stochastic or Doléans-Dade exponential of $X$, denoted by $\mathcal{E}(X)_{t}$ and written explicitly as

$$
\mathcal{E}(X)_{t}=X_{0} e^{X_{t}-\frac{1}{2}[X]_{t}^{c}} \prod_{s \leq t, \Delta X_{s} \neq 0}\left(1+\Delta X_{s}\right) e^{-\Delta Z_{s}} .
$$

Let us now recall a result linking the ordinary and stochastic exponentials of a Lévy process:

Proposition 3. 1. Let $(X)_{t \geq 0}$ be a real valued Lévy process with Lévy triplet $\left(\sigma^{2}, \nu, \gamma\right)$ and $Z=\mathcal{E}(X)$ its stochastic exponential. If $Z>0$ a.s. then there exists another Lévy process $\left(L_{t}\right)_{t \geq 0}$ such that $Z_{t}=e^{L_{t}}$ where

$$
L_{t}=\ln Z_{t}=X_{t}-\frac{\sigma^{2} t}{2}+\sum_{0 \leq s \leq t}\left\{\ln \left(1+\Delta X_{s}\right)-\Delta X_{s}\right\} .
$$

Its Lévy triplet $\left(\sigma_{L}^{2}, \nu_{L}, \gamma_{L}\right)$ is given by:

$$
\begin{aligned}
& \sigma_{L}=\sigma \\
& \nu_{L}(A)=\nu(\{x: \ln (1+x) \in A\})=\int 1_{A}(\ln (1+x)) \nu(d x), \\
& \gamma_{L}=\gamma-\frac{\sigma^{2}}{2}+\int \nu(d x)\left\{\ln (1+x) 1_{[-1,1]}(\ln (1+x))-x 1_{[-1,1]}(x)\right\} .
\end{aligned}
$$

2. Let $(L)_{t \geq 0}$ be a real valued Lévy process with Lévy triplet $\left(\sigma_{L}^{2}, \nu_{L}, \gamma_{L}\right)$ and $S_{t}=\exp L_{t}$ its exponential. Then there exists a Lévy process $(X)_{t \geq 0}$ such that $S_{t}$ is the stochastic exponential of $X: S=\mathcal{E}(X)$ where

$$
X_{t}=L_{t}+\frac{\sigma^{2} t}{2}+\sum_{0 \leq s \leq t}\left\{1+\Delta L_{s}-e^{\Delta L_{s}}\right\} .
$$

The Lévy triplet $\left(\sigma^{2}, \nu, \gamma\right)$ of $X$ is given by:

$$
\begin{aligned}
& \sigma=\sigma_{L}, \\
& \nu(A)=\nu_{L}\left(\left\{x: e^{x}-1 \in A\right\}\right)=\int 1_{A}\left(e^{x}-1\right) \nu_{L}(d x), \\
& \gamma=\gamma_{L}+\frac{\sigma_{L}^{2}}{2}+\int \nu_{L}(d x)\left\{\left(e^{x}-1\right) 1_{[-1,1]}\left(e^{x}-1\right)-x 1_{[-1,1]}(x)\right\} .
\end{aligned}
$$




\section{Appendix B}

Lemma 1. Let $X_{1}$ and $X_{2}$ be random variables with $E\left[\left|X_{i}\right|\right]<\infty, i=1,2$, and denote, by $F_{i}$ the distribution function of $X_{i}$, e.g. $F_{i}(x)=P\left[X_{i} \leq x\right]$, and by $\phi_{i}$ the characteristic function of $X_{i}$. Then

$$
\int_{\mathbb{R}} e^{i u x}\left(F_{1}(x)-F_{2}(x)\right) d x=\frac{\phi_{2}(u)-\phi_{1}(u)}{i u}, \quad u \neq 0 .
$$

In addition, if

$$
\int_{\mathbb{R}} \frac{\left|\phi_{i}(u)\right|}{1+|u|} d u<\infty, \quad i=1,2
$$

then

$$
F_{1}(x)-F_{2}(x)=\frac{1}{2 \pi} \int_{\mathbb{R}} e^{-i u x} \frac{\phi_{2}(u)-\phi_{1}(u)}{i u} d u, \quad \text { all } x .
$$

Proof. First part. Denoting by $p_{i}$ the laws of $X_{i}, i=1,2$, we have

$$
\begin{aligned}
& \int_{\mathbb{R}} e^{i u x}\left(F_{1}(x)-F_{2}(x)\right) d x \\
&=\int_{\mathbb{R}} d x e^{i u x} \int_{\mathbb{R}} d z\left\{1_{x<0} 1_{z \leq x}-1_{x \geq 0} 1_{z>x}\right\}\left(p_{1}(d z)-p_{2}(d z)\right) .
\end{aligned}
$$

From the integrability of $X_{i}$, it follows that

$$
\int_{-\infty}^{0} F_{i}(x) d x<\infty \quad \text { and } \quad \int_{0}^{\infty}\left(1-F_{i}(x)\right) d x<\infty, \quad i=1,2 .
$$

Therefore, we can use the Fubini theorem to interchange the integrals in (12), which produces

$$
\begin{aligned}
& \int_{\mathbb{R}} e^{i u x}\left(F_{1}(x)-F_{2}(x)\right) d x \\
= & \int_{\mathbb{R}} d z\left(p_{1}(d z)-p_{2}(d z)\right)\left\{1_{z \leq 0} \frac{1-e^{i u z}}{i u}-1_{z \geq 0} \frac{e^{i u z}-1}{i u}\right\}=\frac{\phi_{2}(u)-\phi_{1}(u)}{i u}
\end{aligned}
$$

for every $u \neq 0$.

Second part. Without loss of generality, suppose that $F_{2}$ is continuous, because otherwise we could introduce a continuous CDF $F_{3}$ and decompose $F_{1}-F_{2}=F_{1}-F_{3}+\left(F_{3}-F_{2}\right)$. Multiplying both sides of (11) by

$$
\frac{1}{\sigma \sqrt{2 \pi}} e^{-\frac{u^{2}}{2 \sigma^{2}}} e^{-i u t}
$$

and integrating with respect to $u$, we get

$$
\frac{1}{2 \pi} \int_{\mathbb{R}} e^{-\frac{u^{2}}{2 \sigma^{2}}} e^{-i u t} \frac{\phi_{2}(u)-\phi_{1}(u)}{i u} d u=\frac{\sigma}{\sqrt{2 \pi}} \int e^{-\frac{\sigma^{2}}{2}(x-t)^{2}}\left(F_{1}(x)-F_{2}(x)\right) d x .
$$


When $\sigma \rightarrow \infty$, the right-hand side converges to $F_{1}(t)-F_{2}(t)$ in all continuity points of $F_{1}$. By the hypothesis $E\left[\left|X_{i}\right|\right]<\infty, i=1,2, \phi_{i}(u)=1+O(u), i=1,2$, $\frac{\phi_{2}(u)-\phi_{1}(u)}{i u}$ is integrable near zero. Since it is also integrable at infinity by the lemma's condition, the left-hand side converges to

$$
\frac{1}{2 \pi} \int_{\mathbb{R}} e^{-i u t} \frac{\phi_{2}(u)-\phi_{1}(u)}{i u} d u
$$

for every $t$, as $\sigma \rightarrow \infty$, and the proof is completed.

\section{References}

[1] P. Bertrand and J.-L. Prigent, Portfolio insurance: the extreme value approach to the CPPI method, Finance, 23 (2002), pp. 69-86.

[2] F. Black and R. Jones, Simplifying portfolio insurance, Journal of Portfolio Management, 14 (1987), pp. 48-51.

[3] F. Black And A. Perold, Theory of constant proportion portfolio insurance, The Journal of Economics, Dynamics and Control, 16 (1992), pp. $403-426$.

[4] P. CarR And D. Madan, Option valuation using the fast Fourier transform, J. Comput. Finance, 2 (1998), pp. 61-73.

[5] R. Cont and P. Tankov, Financial Modelling with Jump Processes, Chapman \& Hall / CRC Press, 2004.

[6] R. Cont, P. Tankov, and E. Voltchkova, Hedging with options in models with jumps. Proceedings of the 2005 Abel Symposium in Honor of Kiyosi Itô, 2005.

[7] N. El Karoui, M. Jeanblanc, and V. Lacoste, Optimal portfolio management with american capital guarantee, J. Econ. Dyn. Control, (2005), pp. 449-468.

[8] H. Gerber and G. Pafumi, Pricing dynamic investment fund protection, North American Actuarial Journal, 4 (2000), pp. 28-41.

[9] T. Goll and J. Kallsen, Optimal portfolios for logarithmic utility, Stochastic Process. Appl., 89 (2000), pp. 31-48.

[10] S. Kou, A jump-diffusion model for option pricing, Management Science, 48 (2002), pp. 1086-1101.

[11] R. W. LEE, Option pricing by transform methods: extensions, unification and error control, J. Comput. Finance, 7 (2004). 
[12] H. E. Leland And M. Rubinstein, The evolution of portfolio insurance, in Dynamic Hedging: a Guide to Portfolio Insurance, D. Luskin, ed., John Wiley and Sons, 1988.

[13] A. R. Perold, Constant proportion portfolio insurance. Harward Business School, Working paper, 1986.

[14] J.-L. Prigent And F. TAhar, CPPI with cushion insurance. THEMA University of Cergy-Pontoise working paper, 2005.

[15] K. Sato, Lévy Processes and Infinitely Divisible Distributions, Cambridge University Press, Cambridge, UK, 1999.

[16] K. J. Singleton, Estimation of affine asset pricing models using the empirical characteristic function, Journal of Econometrics, 102 (2001), pp. 111-141.

[17] J. YU, Empirical characteristic function estimation and its applications, Econometric Reviews, 23 (2004), pp. 93-123. 